\title{
Desigualdade social e os estudos epidemiológicos: uma reflexão
}

\author{
Social inequality and epidemiological studies: a reflection
}

Maria Angela Fernandes Ferreira ${ }^{1}$

Maria do Rosário Dias de Oliveira Latorre ${ }^{2}$

${ }^{1}$ Programa de Pósgraduação em Saúde Coletiva, Departamento de Odontologia, Centro de Ciências da Saúde,

Universidade Federal do Rio Grande do Norte (UFRN). Av. Rui Barbosa 1257/Bl. A/204, Lagoa Nova. 59056-300 Natal RN. angelaf@ufrnet.br ${ }^{2}$ Programa de Saúde Pública, Departamento de Epidemiologia, Faculdade de Saúde Pública,

Universidade de São Paulo.

\begin{abstract}
Social indicators are now indispensable in the list of variables of epidemiological studies, based on the fact that the determination of health complaints is complex and multidimensional. From this perspective, social inequality has gained prominence as an explanatory factor for the health conditions of populations. The scope of this article is to discuss the different concepts that underpin the selection of the indicators used in epidemiological studies and examine the psychosocial effects on human beings caused by social inequality. A literature review of epidemiological studies that used social inequality and social capital indicators was conducted for a better understanding of health problems, as well as an investigation in the fields of sociology and social psychology. The research revealed that there is some controversy surrounding the effect of social inequality on health, possibly because these indicators are predominantly based on income and individual consumption capacity. Likewise, social capital indicators at cognitive and structural levels are too limited to understand the dynamism of social relations. Accordingly, further studies are needed for the construction of social indicators capable of examining the complexity of modern societies.
\end{abstract}

Key words Epidemiology, Social indicators, Social inequality
Resumo Os indicadores sociais se tornaram imprescindíveis no elenco de variáveis dos estudos epidemiológicos a partir da constatação de que a determinação dos agravos à saúde é complexa e multidimensional. Nessa perspectiva, a desigualdade social vem ocupando destaque como um fator explicativo das condições de saúde das populações. O objetivo é discutir as diferentes concepções que norteiam a seleção dos indicadores utilizados nos estudos epidemiológicos e abordar os efeitos psicossociais nos seres humanos acarretados pela desigualdade social. Foi realizada uma revisão da literatura acerca dos estudos epidemiológicos que utilizaram os indicadores de desigualdade social e capital social para uma melhor compreensão dos problemas de saúde, bem como uma investigação no campo da sociologia e da psicologia social. De acordo com a pesquisa pode-se constatar que há controvérsias sobre o efeito da desigualdade social na saúde humana pelo fato desses indicadores serem baseados, majoritariamente, pela renda e capacidade de consumo dos indivíduos. Da mesma forma, os indicadores de capital social em nível cognitivo e estrutural são muito limitados para compreender o dinamismo das relações sociais. Nesse sentido, são necessários mais estudos para a construção de indicadores sociais que contemplem a complexidade das sociedades modernas.

Palavras-chave Epidemiologia, Indicadores sociais, Desigualdade social 


\section{Introdução}

A influência da desigualdade social sobre a saúde humana tem sido amplamente discutida nos estudos da saúde coletiva. Esse crescente interesse se deve, principalmente, pela constatação de que o crescimento da renda ocorrido em alguns países ricos não diminuiu as suas taxas de mortalidade e nem aumentou a esperança de vida ao nascer, quando comparados a outros ${ }^{1}$. Nesse sentido, uma hipótese levantada é a de que é a desigualdade de renda entre os países e dentro deles que explica tais achados ${ }^{2,3}$.

No entanto, vale salientar que a desigualdade social é uma condição inerente ao próprio sistema capitalista, onde um pequeno grupo de pessoas detém os meios de produção e o capital financeiro, enquanto a grande maioria da população é possuidora apenas de sua força de trabalho. Sendo então a desigualdade um fenômeno socioeconômico, em que medida essa diferença influenciaria a saúde humana? Parece razoável inferir que o efeito deletério sobre a saúde se daria apenas naquelas sociedades com algum grau de iniquidade social. Segundo Whitehead e Dahlgre $^{4}$, a iniquidade seria aquela desigualdade injusta, indesejável e evitável que se funda no caráter essencial da diferença. A iniquidade pressupõe exclusão que é um fenômeno social e cultural, um fenômeno de civilização ${ }^{5}$.

É exatamente na avaliação do efeito da desigualdade que reside a divergência entre os pesquisadores. Para alguns, as taxas de mortalidade e de saúde estão mais relacionadas com as condições materiais - como pobreza absoluta - do que com a desigualdade de renda ${ }^{6,7}$. Para outros, a saúde de um indivíduo é determinada não apenas pela renda individual, mas também pelo cotejamento desta com a média de renda da população em geral, isto é, quanto maior o fosso existente, menor os níveis de saúde ${ }^{8}$.

Quais seriam então as justificativas para a afirmação de que a desigualdade social poderia acarretar problemas à saúde humana? A explicação é psicossocial, ou seja, a partir da percepção que os indivíduos têm do seu lugar na hierarquia social ${ }^{3}$. Os problemas psicossociais causados pela vivência dos indivíduos em sociedades desiguais vêm sendo apontada como uma das grandes responsáveis pelas altas taxas de homicídio, mortalidade e uma menor esperança de vida ao nascer ${ }^{8}$.

A despeito dessa divergência, a introdução da "variável” desigualdade social no elenco das explicativas das investigações epidemiológicas tem sido usada com bastante frequência. O grande desafio dos epidemiologistas, no entanto, é selecionar o melhor indicador para aferir essa desigualdade social. Para tanto, se faz necessário uma boa compreensão da sociedade em que os problemas de saúde ocorrem, o seu modo de produção, suas classes sociais e as relações sociais vigentes (capital social).

Além disso, o conhecimento da natureza das desigualdades sociais e o impacto social de políticas, programas, projetos e ações sobre a saúde e seus determinantes orientam as prioridades para a gestão pública. Nesse sentido, a construção desses indicadores é fundamental para nortear esses gestores na distribuição dos escassos recursos destinados à saúde ${ }^{9}$.

Para a discussão desse tema foi realizada uma revisão sistemática da literatura sobre o efeito da desigualdade social na saúde dos indivíduos. A partir da constatação da controvérsia entre os estudos e das limitações na discussão sobre os efeitos psicossociais da desigualdade social, foram pesquisados livros vinculados ao campo da sociologia e psicologia que pudessem esclarecer essas questões.

Diante dessa exposição inicial, o objetivo deste artigo é discutir as diferentes concepções que norteiam a seleção dos indicadores utilizados nos estudos Epidemiológicos e abordar os efeitos psicossociais no ser humano acarretados pela desigualdade social.

\section{Que concepções norteiam a seleção dos indicadores em saúde?}

$\mathrm{Na}$ área da saúde, diversos estudos apontam uma significativa associação entre a condição socioeconômica e os agravos à saúde. A partir da constatação de que existem desigualdades no perfil de saúde nos diversos grupos e ambientes sociais, então, como aferir a influência desses efeitos sociais sobre a saúde dos indivíduos? Seria através dos indicadores de renda e de atributos individuais e/ou de indicadores populacionais ou contextuais?

Aqueles que consideram a saúde como sendo uma condição de responsabilidade unicamente do indivíduo, cujos hábitos são decorrentes de escolhas e responsabilidade pessoais, utilizam indicadores exclusivamente individuais. Nos estudos Epidemiológicos, os indicadores socioeconômicos como renda, grau de escolaridade, ocupação, condições de moradia, além de outros proxys, são comumente utilizados naqueles individuados.

Por outro lado, os que defendem que a aquisição de hábitos deletérios a saúde depende do 
contexto em que tais populações vivem e que são determinados pela sua história de vida e das condições estruturais do ambiente (acesso às políticas públicas, por exemplo), optam pelos indicadores contextuais (Índice de capital social da área, Índice de dissimilaridade, Índice de desigualdade, violência e outros índices compostos).

O primeiro entendimento está baseado na perspectiva de que a diferenciação socioeconômica entre as pessoas está associada ao valor humano fundamental como a liberdade e o desempenho das potencialidades individuais, das oportunidades que ele soube aproveitar. A aferição da saúde, portanto, seria baseado na quantificação de seus bens e na sua capacidade de consumo, o que lhe proporcionaria uma melhor condição de saúde. A segunda concepção defende que essa diferença social está relacionada com a exploração e a injustiça social e os comportamentos negativos são consequência dessa diferença, ou seja, das privações materiais, que são a causa e a consequência das dificuldades em obter uma melhor condição de saúde ${ }^{10}$.

Ainda nessa linha de discussão sobre o efeito da renda individual ou a relativa, Kawachi et al. ${ }^{11}$ identifica a existência de duas hipóteses - Hipótese da renda absoluta (HRA) e Hipótese de Renda Relativa (HRR). De acordo com a HRA, o estado de saúde de um indivíduo depende apenas da sua renda individual e desde que sua renda seja adequada para suas necessidades básicas, então ele teria uma boa condição de saúde. Em relação à outra hipótese, a da renda relativa (HRR), as condições de saúde não dependem apenas das condições materiais de vida, mas também da posição relativa que cada pessoa ocupa na sociedade.

Após uma reflexão sobre os indicadores mais adequados para a aferição da desigualdade em saúde, Magalhães ${ }^{12}$ destaca as limitações da renda como variável explicativa para o entendimento dos perfis epidemiológicos. Ressalta ainda a preocupação com a comparação entre os indicadores de saúde de grupos menos favorecidos com a média da população em geral, na medida em que tende a mascarar as diferenças relativas entre os grupos sociais.

Diante dessa controvérsia é importante ressaltar que alguns estudos demonstram que quando os níveis de renda e as necessidades básicas ultrapassam a linha da pobreza, a renda individual por si só não explica as desigualdades em saúde ${ }^{2,13}$. Isso favoreceria a ideia de que os indicadores referentes à HRR poderiam ser mais sensíveis na análise da desigualdade em saúde.
Como afirmado anteriormente, na concepção da HRR, a saúde do indivíduo é medida pela distância relativa da sua renda e da média da população. Mas será que os indivíduos têm consciência de sua posição hierárquica na sociedade? Acredita-se que desde criança já é possível perceber as diferenças sociais pela ocupação, casa, carros, etc. e fazer comparações sociais indesejáveis ${ }^{14}$. De acordo com Pickett e Wilkinson ${ }^{15}$, a desigualdade de renda está fortemente associada ao bem estar da criança e, portanto, a melhoria das condições de saúde em países ricos depende mais da redução das desigualdades que do crescimento econômico.

\section{A despeito dessa divergência, os indicadores de renda utilizados nos estudos epidemiológicos são os mais adequados para aferir desigualdade social?}

A resposta a essa questão reflete novamente a visão de mundo do pesquisador. A visão puramente econômica de compreender o mundo, chamada de "economicista" por Souza ${ }^{16}$, é superficial, na medida em que mascara os "valores imateriais" que são transferidos pelas classes sociais e que mantêm os privilégios da classe média e alta ao longo do tempo.

O economicismo liberal, assim como o Marxismo tradicional, percebe a realidade das classes apenas economicamente, no primeiro caso como produto da renda e no segundo como lugar na produção. Dessa forma, esconde e torna invisível todos os fatores e precondições sociais, emocionais, morais e culturais que constituem a renda diferencial, confundindo causa e efeito.

No Brasil, por exemplo, um índice bastante popular na aferição da renda individual é o ABA/ ABIPEMA (Associação Brasileira de Anunciantes - Associação Brasileira de Institutos de Pesquisa de Mercado), que objetiva mensurar o poder aquisitivo dos consumidores (itens de consumo - rádio, TV, etc., presença de empregada doméstica e nível de educação) e que divide a sociedade em cinco classes sociais - A, B, C, D, E. Como pode ser observado, esse índice não foi desenvolvido para aferir condições de saúde e bem-estar social. Além disso, hábitos de consumo não necessariamente refletem padrões comportamentais em saúde, níveis diferenciais de acesso a serviços médicos ou risco de doença ${ }^{10}$.

Outros indicadores, tais como, o nível educacional, medidos em graus ou em números de anos, também é bastante utilizado nos estudos epidemiológicos. Isso porque, de alguma forma, 
o grau de escolaridade prediz a renda e a ocupação dos indivíduos. Atualmente, a preocupação entre os pesquisadores é conhecer a qualidade da educação recebida, já que para a saúde a qualificação, o grau de informação, o conhecimento e a habilidade de cognição são essenciais. Em relação à variável ocupação do "chefe da família" para aferir o status socioeconômico, a pressuposição é que a mesma está relacionada a renda, status social, rede social, stress, autonomia, salubridade, além de outras questões que podem interferir na saúde dos indivíduos.

Outra informação bastante coletada nesses estudos são as condições da habitação, usada para avaliar a situação da casa, quantidade de pessoas por cômodo, número de banheiros, vizinhança $\mathrm{e}$ bairro. A partir desses dados espera-se aferir os diversos estratos sociais. No entanto, há uma grande dificuldade de comparação com outros estudos, já que esses dados são específicos de cada lugar.

Existem ainda vários indicadores compostos para a aferição da posição socioeconômica. Dentre estes, destacam-se os index Cambridge Social Interaction and Stratification Scale (CAMSIS), Carstairs Deprivation, Deprivation, Registrar General's Social Classes (RGSC), Hollingshead of social position, Duncan, Nam-Powers socioeconomic status, Warner's index of status characteristics, entre outros ${ }^{17}$. No nível ecológico, identificam-se os índices de Townsend deprivation, Jarman ou underprivileged área (UPA) score e Breadline Britain.

Nas investigações que objetivam avaliar o efeito das desigualdades sociais sobre a saúde, comumente, são usados indicadores baseados na renda da população, como por exemplo, os coeficientes de GINI e Theil, Índice de Robin Wood e Atinkson entre diversos com origem puramente estatística. Estes índices são baseados em medidas de dispersão, como desvio padrão, amplitude, distância inter-quartis, e coeficientes de variação de renda entre pobres e ricos ${ }^{17}$.

Como pode ser observado, a grande maioria dos indicadores sociais utilizados nos estudos epidemiológicos estão pautados na quantidade de renda e nos bens dos indivíduos. Entretanto, para compreender o fenômeno da desigualdade social, é necessário entender a sua gênese e a sua reprodução no tempo. De acordo com Souza ${ }^{16}$, são o "capital cultural" e o "capital econômico" que constituem toda a hierarquia social e permitem a reprodução da sociedade moderna. $\mathrm{O}$ "capital cultural", representado pelo conhecimento técnico e escolar, historicamente apropriado pe- las classes médias, que se constitui em uma das classes dominantes desse tipo de sociedade. Já a classe alta se caracteriza pela apropriação do "capital econômico", ainda que alguma porção do "capital cultural" esteja presente.

Os valores inerentes ao chamado "capital cultural" da classe média, na concepção de Souza ${ }^{16}$, não aferidos pelos indicadores de renda, são aqueles ensinados nas famílias, quais sejam: a disciplina, o autocontrole, o pensamento prospectivo, o respeito ao espaço alheio, etc. Segundo o autor, esse aprendizado é transmitido afetiva e silenciosamente no refúgio dos lares. Tais características emocionais e cognitivas irão, mais tarde, permitir o sucesso na escola e no mercado de trabalho. Portanto, a renda econômica advinda desse sucesso é efeito e não causa das diferenças entre as classes.

Os filhos dessa classe se habituam, desde a tenra idade, a verem os pais lendo, os tios falando um idioma, os irmãos desvendando os segredos do computador, os amigos e os familiares falando sobre os encantos artísticos das cidades europeias, além do acesso às relações familiares privilegiadas, etc., ou seja, existe todo um ambiente familiar propício ao estudo, em cujo processo de identificação afetiva se dá de modo natural e eficaz. Essa herança da classe média, imaterial por excelência, é completamente invisível para a visão economicista dominante, que universaliza os pressupostos da classe média para todos como se as condições devidas fossem as mesmas.

Excluída desses "capitais impessoais" e do processo de modernização brasileiro, está a "ralé", provocativamente denominada por Souza ${ }^{16}$, constituída por todos aqueles excluídos e abandonados, que se reproduzem há gerações enquanto tal. No ambiente da "ralé" brasileira é comum os maus-tratos das crianças, a naturalização do estupro, o alcoolismo, enfim, a desagregação familiar que provocam danos emocionais e cognitivos irreparáveis, afetando a autoestima e dificultando enormemente a sua formação enquanto sujeito para competir com sucesso no mercado de trabalho.

Como esperar dos pais dessa classe, o exemplo de valor ao estudo, se eles não tiveram essa oportunidade, não foram à escola ou não tiveram a experiência pessoal de sucesso escolar? Assim sendo, por não conhecerem de modo emotivo e por vivência própria os benefícios da vida escolar, dificilmente terão condições de darem o exemplo de estudo, da leitura e da moral. Sem as precondições sociais e familiares, portanto, raramente um membro dessa classe alcançará o 
sucesso escolar e profissional, uma vez que é falsa a ideia de que o mérito é de responsabilidade única do indivíduo.

Nesse sentido, o "capital cultural", na sociedade moderna, passa a marcar a fronteira entre o fracasso e o sucesso social para todas as classes e indivíduos, na luta pelos recursos escassos, materiais e ideias desse tipo de sociedade. Essa forma individualizada de desigualdade, construída para negar a forma real e efetiva da produção classística da desigualdade, é exatamente a "ideologia da meritocracia”. Segundo essa ideologia, a desigualdade é justa e legítima na medida em que reflete o mérito diferencial dos indivíduos. Ideologicamente, essa concepção é aceita e legitimada pela sociedade, descontextualizando o ambiente social no qual os órfãos do "capital cultural" estão inseridos, razão por que as oportunidades lhes são subtraídas ${ }^{16}$.

Considerando, portanto, que os

indicadores econômicos não são

suficientes para aferirem as desigualdades

em saúde e que a compreensão das

relações familiares e sociais são essenciais na explicação das condições de saúde, quais seriam os indicadores complementares mais apropriados para

os estudos Epidemiológicos?

Nos estudos epidemiológicos, o indicador mais utilizado para a aferição das relações sociais é o chamado capital social ${ }^{18}$. Putnam ${ }^{19}$, um dos pioneiros nos estudos sobre capital social, afirma que este se reflete no grau de confiança existente entre os diversos atores sociais, seu grau de associativismo e o acatamento às normas de comportamento cívico, tais como o pagamento de impostos e os cuidados com que são tratados os espaços públicos e os "bens comuns". Segundo o autor, em vez de controles e relações de dominação patrimonialistas, o capital social favorece o funcionamento de normas e sanções consentidas, ressaltando os interesses públicos coletivos.

Nesse sentido, a construção de capital social repercute favoravelmente na coesão da família, da comunidade e na sociedade. Essa coesão social que resulta de uma comunidade participativa é reconhecida como um importante instrumento para o desempenho político e o funcionamento da democracia.

Há uma preocupação em relação às diferenças conceituais de capital social e se esses verdadeiramente facilitam o entendimento sobre a influência das relações comunitárias na saúde. A com- plexidade crescente desses enfoques conceituais dificulta a construção de instrumentos capazes de operacionalizá-los. De acordo com Souza e Grun$\mathrm{dy}^{20}$, a utilização do conceito de capital social exige cautela, a fim de que o seu uso não seja um pretexto para esconder ideologias indesejadas ou perpetuar o "status quo" de indivíduos ou grupos com interesses políticos ou econômicos específicos. Por isso, os autores recomendam o exame cuidadoso do conceito de capital social norteadores dos trabalhos existentes na literatura.

Fatores adversos à formação de capital social são a desigualdade na distribuição da renda e de oportunidades, o desemprego e as catástrofes naturais que levam às migrações, desarticulando a rede de relações sociais existentes e exigindo dos indivíduos grandes esforços nas tentativas de reconstrução de uma rede de relações sociais de apoio e confiança. As maiores vítimas no processo de desestruturação são as crianças, particularmente, as de famílias desorganizadas, que apresentam baixos índices de aprendizagem e elevadas taxas de evasão escolar ${ }^{21}$.

Segundo Hirschman ${ }^{22}$, o capital social não se desgasta com o uso e não se esgota, mas pode ser destruído ou reduzido, aumentando a vulnerabilidade dos mais pobres e mais fracos, dos desempregados e desabrigados sujeitos às manifestações das diferentes formas de violência, agressões e delinquência, transformando o ambiente numa situação em que o homem se torna o lobo dos outros ("homo homini lupus").

Nessa perspectiva, o Estado tem um papel fundamental na criação do capital social. O grau de confiança geral e as normas de cooperação social melhoram significativamente com os avanços no desenvolvimento econômico e com políticas de distribuição de renda. Como hipótese a ser testada empiricamente, afirma-se que quanto menor a polarização entre ricos e pobres, maior o capital social, maior a participação em associações e projetos coletivos, maior a renda e melhores as práticas produtivas, na agricultura e na indústria. Da mesma forma, quanto maior a cooperação com a administração pública melhor a qualidade dos serviços públicos e maior a influência no rendimento das crianças nas escolas e a eficiência dos serviços de saúde ${ }^{23}$.

No que se refere aos instrumentos de aferição do capital social utilizados nos estudos da área da saúde, identificam-se aqueles aplicados em níveis individual e coletivo. Isso porque o capital social pode ser investigado através de duas dimensões - cognitiva (individual) e estrutural (contextual). A cognitiva é a esfera onde se avalia 
a percepção individual do nível de confiança interpessoal, ou seja, a capacidade de relacionamento, a rede de contatos sociais baseada em expectativas de reciprocidade e comportamento confiáveis que, no conjunto, melhoram a eficiência individual. Em relação à saúde significaria a confiança nas normas de comportamento, através do controle de hábitos deletérios, prestação de ajuda e apoio mútuo e os meios informais de troca de informação ${ }^{24}$.

Por outro lado, o componente estrutural mede, em nível coletivo, a coesão social, pela obediência às normas e leis; a negociação em situação de conflito e a predominância da cooperação sobre a competição, o que resultaria em um estilo de vida baseado na associação espontânea, no comportamento cívico, enfim, numa sociedade mais aberta e democrática ${ }^{25}$. De uma maneira geral, os estudos agregam as duas dimensões, seja utilizando dados individuais ou agregados.

Após uma análise dos estudos sobre capital social e saúde encontrados na literatura, pode-se observar que grande parte dos dados coletados para a aferição desse indicador são oriundos de censos $^{26,27}$ ou inquéritos nacionais ${ }^{28,29}$. Dessas pesquisas são subtraídas informações acerca da confiança nos amigos, na família e membros da comunidade e a participação em organizações sociais (religiosa, cultural, serviços sociais, esportiva, artística, política e outras). Entre os dados contextuais se encontram a confiança no governo e suas instituições, a coesão social (taxas de divórcio, desemprego, crimes e conflitos no trabalho), número de espaço público e organizações voluntárias per capita, entre outros.

No entanto, alguns instrumentos foram construídos especificamente com a finalidade de aferir capital social, dentre estes o Questionário Integrado para Medir Capital Social (QI-MCS) produzido pelo Banco Mundial em 2003. Esse questionário contempla aspectos como: os grupos e redes existentes na comunidade, a confiança e a solidariedade e a ação coletiva e nível de cooperação social. Além disso, verifica a rede de informação e comunicação, coesão e inclusão social e a autoridade e organização política da comunidade ${ }^{30}$.

Em uma revisão sistemática sobre o efeito do capital social e os indicadores de saúde, Islam et al. ${ }^{18}$ concluíram que existe uma forte correlação entre saúde e capital social. No entanto, quando os dados foram avaliados através de análise multinível, essa associação só foi verificada nas sociedades economicamente desiguais, significando que em regiões com menor desigualdade so- cial não se consegue detectar o efeito do capital social. De acordo com os autores, a maior dificuldade na análise dos diversos estudos incluídos (42 estudos) são as diferenças nas concepções de capital social refletidas nos mais diversos indicadores e as limitações metodológicas dos desenhos de estudo.

Vale destacar a pertinência da análise multinível nesses estudos, na medida em que os efeitos contextuais, inerentes à dimensão estrutural do capital social (estado/comunidade/vizinhança), necessitam ser avaliados em um nível superior. Essa análise permite-nos determinar se as características da área em que os indivíduos residem afetam a saúde individual ${ }^{31}$.

Os indicadores de capital social, no entanto, são criticados por alguns que enfatizam a importância dos regimes políticos, da ideologia e das instituições como os grandes responsáveis pela saúde da população $0^{32,33}$. Além disso, verifica-se nesses estudos a ausência da discussão sobre as relações de classe social, que é o determinante mais importante para explicação da saúde da população.

Como pode ser observado, há muitas dificuldades em se construir modelos explicativos mais complexos que contemplem todas as dimensões do processo saúde-doença. O importante é reconhecer os limites, as ambiguidades e as lacunas existentes e se buscar esforços analíticos-descritivos no sentido de se contrapor aos modelos explicativos simplistas e lineares ${ }^{12}$.

\section{Considerando que a desigualdade social produz consequências sobre a saúde da população, então, que efeitos psicossociais são esses?}

A comprovação de que a desigualdade social acarreta problemas mentais foi obtida entre os países desenvolvidos, através de estudos ecológicos e individuados. Foi observado que a alta prevalência de doenças mentais está correlacionada com o aumento da renda per capita nacional ${ }^{34} \mathrm{e}$ baixo capital social em adultos ${ }^{24}$.

A explicação plausível para tal consiste no fato da desigualdade social provocar efeitos psicossociais como humilhação e perda de autoestima. Nesse sentido, em condições de relativa privação socioeconômica esses indivíduos sofreriam de stress crônico que direta ou indiretamente afetaria a sua saúde ${ }^{11}$.

Os efeitos psicossociais mais evidentes em uma sociedade de classes é a humilhação social. Esse é um sofrimento longamente aturado e "rumina- 
do" pelas pessoas da classe pobre. É um sentimento ancestral e repetido em função da violência material e psicológica sofrida por várias gerações, iniciada através de golpes de espoliação e servidão. É uma dor que uma multidão de pessoas subordinadas sente que esvazia a alma e fere marcando indelevelmente o espírito mais resistente ${ }^{35}$.

Uma sociedade com fortes características de iniquidades proporcionam as pessoas hierarquicamente inferiores atos de humilhação pública que age por dentro provocando uma angustia profunda. Tal angústia é o sentimento recorrente entre os excluídos e o mais abstrato e desqualificado dos afetos. É a emoção mais comum entre os humanos. De acordo com Costa ${ }^{35}$, tais grupos populacionais se sentem invisíveis socialmente, porque não são percebidos pelos demais. Essa indiferença é traduzida em "lágrimas, gagueira, emudecimento, endurecimento ou agitação do corpo, o protesto confuso, a ação violenta e até mesmo o crime".

Ainda segundo Costa ${ }^{35}$, a invisibilidade pública é o desaparecimento intersubjetivo de um homem no meio dos outros e expressão de dois fenômenos psicossociais nas sociedades capitalistas - humilhação social e reificação. A humilhação social é expressão da desigualdade política, indicando exclusão subjetiva de uma classe inteira de homens. Tal exclusão política "fabrica sintomas, infestando o afeto, o raciocínio, a ação e o corpo do homem humilhado". Tem um poder nefasto na medida em que molda a subjetividade do indivíduo pobre tolhendo a sua capacidade de criar, esvaziando assim as condições que possibilitariam transcender a compreensão imediata e estática da realidade.

A experiência da humilhação é uma realidade inapelável, "fincado na carne, vivida como uma espécie de queimadura: arde marca-lhe o corpo como cicatriz, rouba-lhe sensibilidade, torna corpo e alma vulneráveis, frágeis. A experiência de ser humilhado fere a percepção de si mesmo, fere a estima por si própria: a imagem refletida no outro com distorção"35.

A reificação é também um processo histórico de longa duração através do qual as sociedades modernas fundaram seus alicerces sob o princípio das determinações mercantis. Nesse sentido, a reificação configura-se como processo pelo qual, nas sociedades capitalistas, o valor das pessoas, das relações inter-humanas, dos objetos e das instituições apresentam-se as consciências dos homens como valor econômico, valor de troca. Tudo se torna mercadoria. A sociedade que vive à custa desse mecanismo produz e reproduz, per- petua e apresenta relações sociais como relação entre coisas. Assim, "o homem fica apagado, mantido à sombra. A cegueira de gente que não enxerga gente é traumática, causa angústia e dispara humilhação”. A experiência de não aparecer como gente estando no meio de gente é um fenômeno psicossocial. O homem tornado invisível, publicamente invisível, sente-se muito mal com isso, "fica murcho, como que faleceu, uma espécie de morte" 35 .

Na verdade, essa violência "simbólica” é atualizada cotidianamente, por todos nós, nos mais “inocentes” encontros casuais, até a "má-fé” institucional generalizada, aceita e legitimada por todos. É o abandono social de toda uma classe, objetivamente percebida como "animalizada", que é excluída e aceita na prática social sem nenhuma perplexidade ou indignação. É por serem percebidos como meros "corpos", numa sociedade que valoriza a disciplina e o autocontrole, é que essa classe desprezada é vista como perigosa e como assunto de "polícia" e não de "política"16.

A segregação de espaços sociais é outro aspecto que exprime materialmente a humilhação de lugares reservados e lugares vetados, lugares prestigiados e lugares indigentes, lugares de ricos e lugares de pobres. O fato dos pobres e excluídos não recusarem a segregação deve-se à nefasta informação da ideologia; informações que mascaram os motivos históricos e forjam explicações apaziguadoras pelos quais uma classe inteira de homens está a alimentar uma outra.

Os fatores psicossociais que atuam precocemente no curso da vida influenciam grande número de variáveis biológicas. Apesar das evidências ainda não serem conclusivas, há plausibilidade biológica para explicar o efeito dos fatores psicossociais na saúde da população. Após exaustiva revisão de literatura sobre o assunto, Brunner ${ }^{36}$ concluiu que o estresse sobre a história de acumulação de experiências psicossociais a partir de infância e continuando ao longo da vida parece ter efeitos biológicos que irão influenciar o desenvolvimento de doenças degenerativas. Segundo o autor, mecanismos de estresses neuroendócrinos podem contribuir, particularmente, em gradientes sociais no risco de doença coronária e morbidade associada com a imunidade reduzida. Afirma ainda que a distribuição social observada de doenças crônicas representa um desafio para a compreensão dos processos fisiopatológicos, que têm lugar ao longo de muitos anos e envolvem muitos sistemas do corpo.

A perspectiva biológica, no entanto, não diminui a importância primordial da organização 
social na geração de desigualdades em saúde, mas pode proporcionar uma abordagem para determinar a aspectos específicos do ambiente psicossocial que influenciam a saúde.

\section{Considerações finais}

A partir das reflexões expostas neste texto, podese constatar que há controvérsias quanto ao efeito da desigualdade social na saúde dos indivíduos. Alguns defendem que é a baixa renda ou a pobreza absoluta que interferem sobre as péssimas condições de saúde, enquanto outros acreditam que, além disso, a posição de inferioridade que alguns ocupam na hierarquia social também produzem efeitos psicossociais deletérios sobre os indivíduos. Tais efeitos são decorrentes da humilhação ou até da completa "invisibilidade" daqueles que se encontram em uma posição hierárquica inferior, bem como em função das relações inter-humanas comercializadas (reificação) impostas pela sociedade capitalista moderna.

Pode-se observar ainda que o conceito de desigualdade social é multidimensional, ou seja, são muitos os aspectos que devem ser analisados para que seja possível a aferição dessa "variável" e que, em função disso, há grandes limitações dos indicadores atuais em contemplarem toda essa complexidade conceitual. Isso porque esses indicadores foram construídos, na sua maioria, a partir da renda e da capacidade de consumo dos indivíduos, não contemplando os chamados "valores imateriais" que determinam fortemente a constituição das classes sociais.

As lacunas dos indicadores baseados apenas na renda dos indivíduos são preenchidas parcialmente pelas informações obtidas a partir das relações sociais verificadas em uma determinada sociedade - o chamado capital social. Os indicadores de capital social apreendem informações acerca dos aspectos cognitivos e estruturais das relações entre os indivíduos e as suas redes sociais, no âmbito pessoal e contextual. Entretanto, pode-se constatar uma grande variedade de indicadores contextuais (coesão social), dificultando a comparação entre os estudos, e uma limitação de informações que contemplem a complexidade das relações sociais.

Finalmente, pode-se concluir que o mais importante é o pesquisador compreender as diversas concepções conceituais que norteiam a construção dos indicadores epidemiológicos e usálos de forma crítica compreendendo que nenhum deles consegue abranger a complexa dimensão do processo saúde-doença.

\section{Colaboradores}

MAF Ferreira escreveu o artigo e MRDO Latorre o revisou. 


\section{Referências}

1. Wilkinson RG. Income distribution and life expectancy. BMJ 1992; 304(6820):165-168.

2. Marmot M, Wilkinson RG. Psychosocial and material pathways in the relation between income and health: a response to Lynch et al. BMJ 2001; 322(7296): 1233-1236.

3. Wilkinson RG. Socioeconomic determinants of health. Health inequalities: relative or absolute material standards? BMJ 1997; 314(7080):591-595.

4. Whitehead M, Dahlgre G. The concept and principles of equity and health: Levelling up Part I. Copenhagen: Pan American Health Organization (CID/ HSP/PAHO); 1991.

5. Santos BS. A construção Multicultural da Igualdade e da Diferença. [Publicação Seriada do Centro de Estudos Sociais] 1999 Jan. 61p. [acessado 2012 ago 9]. Disponível: http://www.ces.uc.pt/publicacoes/oficina/135/135.pdf

6. Lynch J, Smith GD, Harper S, Hillemeier M, Ross N, Kaplan GA, Wolfson M. Is income inequality a determinant of population health? Part 1. A systematic review. Milbank Q. 2004; 82(1):5-99.

7. Smith DG, Egger M. Commentary: Understanding It All-Health, Meta-Theories, and Mortality Trends. BMJ 1996; 313(7072):1584-1585.

8. Wilkinson RG, Pickett KE. Income inequality and population health: A review and explanation of the evidence. Soc Sci Med 2006; 62(7):1768-1784.

9. Drachler ML, Côrtes SMV, Castro JD, Leite JCC. Proposta de metodologia para selecionar indicadores de desigualdade em saúde visando definir prioridades de políticas públicas no Brasil. Cien Saude Colet 2003; 8(2):461-470.

10. Antunes JLF. Socioeconomic status and health: a discussion of two paradigms. Rev Saude Publica 2008; 42(3):1-6.

11. Kawachi I, Subramanian SV, Almeida-Filho N. A glossary for health inequalities. J Epidemiol Community Health 2002; 56(9):647-652.

12. Magalhaes R. Monitoramento das desigualdades sociais em saúde: significados e potencialidades das fontes de informação. Cien Saude Colet 2007; 12(3): 667-673.

13. Marmot M. The influence of income on health: views of an Epidemiologist. Health Aff (Milwood). 2002; 21(2):31-46.

14. Backett-Milburn K, Cunningham-Burley S, Davis J. Contrasting lives, contrasting views? Understandings of health inequalities from children in differing social circumstances. Soc Sci Med. 2003; 57(4): 613-623.

15. Pickett KE, Wilkinson RG. Child wellbeing and income inequality in rich societies: ecological cross sectional study. BMJ 2007; 335(7629):1080.

16. Souza J. A Ralé Brasileira: quem é e como vive. Belo Horizonte: Ed. UFMG; 2009.

17. Shaw M, Galobardes B, Lawlor DA, Lynch J, Wheeler B, Smith GD. The Handbook of inequality and socioeconomic position-Concepts and measures. Bristol: Ed. The Policy Press; 2007.

18. Islam MK, Merlo J, Kawachi I, Lindström M, Gerdtham UG. Social capital and health: Does egalitarianism matter? A literature review. Int J Equity Health 2006; 5(3):1-28
19. Putnam RD. Democracies in Flux: The Evolution of Social Capital in Contemporary Society. New York: Oxford University Press; 2002.

20. Souza EM, Grundy E. Promoção da Saúde, Epidemiologia e Capital Social. Cad Saude Publica 2004; 20(5):1354-1360.

21. Rattner H. Prioridade: construir o capital social. Rev Espaço acadêmico 2003; 21:1-8.

22. Hirschman AO. Against parsimony. Am Econ Rev 1984; 74(2):89-96.

23. Kliksberg B. Falácias e Mitos do Desenvolvimento Social. S.Paulo: Cortez Editora, UNESCO; 2001.

24. De Silva MJ, McKenzie K, Harpham T, Huttly SRA. Social capital and mental illness: a systematic review. J Epidemiol Community Health 2005; 59(8):619627.

25. Coleman JS. Social capital in the creation of human capital. Am J social 1988; 94(Supl.):95-120.

26. Kawachi I, Kennedy B, Lochner K, Prothrow-Stith D: Social Capital, Income Inequality, and Mortality. Am. J Public Health 1997; 87(9):1491-1498.

27. Wilkinson RG, Kawachi I, Kennedy BP. Mortality, the social environment, crime and violence. Social Health Illness 1998; 20(5):578-597.

28. Drukker M, Buka SL, Kaplan C, McKenzie K, Van OSJ. Social capital and young adolescents' perceived health in different sociocultural settings. Soc Sci Med 2005; 61(1):185-198.

29. Kennelly B, O'Shea E, Gavey E. Social Capital, life expectancy and mortality: a cross-national examination. Soc Sci Med 2003; 56(12):2367-2377.

30. Grootaert C, Narayan D, Jones VN, Woolcock M. Questionário Integrado para Medir Capital Social (QIMCS). [Publicação Banco Mundial] 2003. Jun. 73p. [acessado 2012 ago 12]. Disponível em: http:// empreende.org.br/pdf/Capital\%20Social\%20e\% 20Cidadania/Question\%C3\%A1rio\%20Integrado \%20para\%20Medir\%20Capital\%20Social.pdf

31. Celeste RK, Nadanovsky P. Aspectos relacionados aos efeitos da desigualdade de renda na saúde: mecanismos contextuais. Cien Saude Colet 2010; 15(5): 2507-2519.

32. Muntaner C, Lynch J. Income inequality, social cohesion, and class relations: A critique of Wilkinson's neo-Durkheimian research program. Int $J$ Health Serv. 1999; 29(1):59-81.

33. Navarro V. A critique of social capital. Int $J$ Health Serv. 2002; 32(4):424-432.

34. Pickett KE, James OW, Wilkinson RG; Income inequality and the prevalence of mental illness: a preliminary international analysis $J$ Epidemiol Community Health. 2006; 60(7):646-647

35. Costa FB. Homens Invisiveis: relatos de uma humilhação social. São Paulo: Ed. Globo; 2008.

36. Brunner E. Stress and the biology of inequality $B M J$ 1997; 314(7092):1472-1476.

Artigo apresentado em 16/05/2011

Aprovado em 19/07/2011

Versão final apresentada em 06/08/2011 